\title{
Paediatric massage for treatment of acute diarrhoea in children: a meta-analysis
}

\author{
Li Gao, Chunhua Jia ${ }^{*}$ (D) and Huiwen Huang
}

\begin{abstract}
Background: Massage therapy has been used by many traditional Chinese medicine physicians to treat acute diarrhoea in children. Since no relevant systematic reviews assessed the clinical effectiveness or the risk of massage therapy, in this study, a meta-analysis was conducted to evaluate the efficacy of paediatric massage for the treatment of acute diarrhoea in children.

Methods: In this meta-analysis, paediatric patients who were diagnosed with acute diarrhoea were included. Interventions using massage therapy alone or combined with other non-pharmacological approaches were included, while in the control groups, patients received pharmacotherapy. The primary outcome was clinical effective rate. Seven databases were used in our research, and the following search terms were used: (massage OR tui na OR manipulation OR acupressure) AND (infant OR child OR baby OR paediatrics) AND (diarrhoea OR diarrhoea) AND (randomized controlled trial). The search date was up to April 30, 2018.

Results: A total of 26 studies encompassing 2644 patients were included in this meta-analysis. It was shown that paediatric massage was significantly better than pharmacotherapy in treating acute diarrhoea in children in terms of clinical effective rate $(n=2213, \mathrm{RR}=1.20,95 \% \mathrm{Cl}: 1.14$ to 1.27$)$, clinical cure rate $(n=345, \mathrm{RR}=1.37,95 \%$ Cl: 1.19 to 1.57$)$, and cure time ( $n=513, \mathrm{MD}=-0.77,95 \% \mathrm{Cl}:-0.89$ to -0.64$)$. However, the quality of evidence for this finding was low due to high risk of bias of the included studies.
\end{abstract}

Conclusions: The present work supported paediatric massage in treating acute diarrhoea in children. More welldesigned randomized controlled trials are still needed to further evaluate the efficacy of paediatric massage.

Keywords: Massage therapy, Children, Acute diarrhoea, Meta-analysis

\section{Background}

Acute diarrhoea is a common disease in children in developing countries, especially for those younger than five years old [1]. There are many causes for acute diarrhoea in children [2], such as viruses or bacterial infection, malabsorption, and inflammatory bowel disease. Delayed treatment may cause dehydration, electrolyte imbalance, or even death. Currently, the main treatment for acute diarrhoea is pharmacotherapy, such as oral rehydration salts, Zinc supplement [3], probiotics, or loperamide [4].

In addition to pharmacotherapy, in China, massage therapy is also used by many traditional Chinese medicine (TCM) physicians to treat acute diarrhoea in children. Massage therapy is defined as the manipulation

\footnotetext{
* Correspondence: chjia11@163.com

Beijing University of Chinese Medicine, No. 11 North 3rd Ring East Road, Chaoyang District, Beijing 100029, China
}

of the soft tissue of the body, and it is part of the complementary and alternative medicine. Most commonly, massage therapy is conducted on meridians and acupuncture points. The theory behind this therapy was outlined in Huangdi Neijing, which is an ancient Chinese medical book. Over the centuries, massage therapy has been used for emotional and physical healing $[5,6]$. There are many benefits of massage therapy [7-10], such as enhancing immune function, unblocking meridians and collateral, activating Qi and blood, and improving the flow of Qi through the meridians. As a result, self-healing in the body is promoted. Paediatric massage is the massage therapy for children that aims to promote health $[8,11]$.

Many studies have assessed the effects of massage therapy. Vickers et al. [12] conducted a meta-analysis to assess the effects of massage therapy for improving

(c) The Author(s). 2018 Open Access This article is distributed under the terms of the Creative Commons Attribution 4.0 International License (http://creativecommons.org/licenses/by/4.0/), which permits unrestricted use, distribution, and reproduction in any medium, provided you give appropriate credit to the original author(s) and the source, provide a link to the Creative Commons license, and indicate if changes were made. The Creative Commons Public Domain Dedication waiver (http://creativecommons.org/publicdomain/zero/1.0/) applies to the data made available in this article, unless otherwise stated. 
health and development in preterm birth and low birth weight infants. The results showed that infants who received massage therapy demonstrated improved weight gain $(5 \mathrm{~g} / \mathrm{d})$ and shorter hospital stays (4-5 days) compared to control groups who did not receive daily massage. Beider et al. [13] conducted a review to examine the effectiveness of massage therapy, and it was shown that massage therapy has real value to the paediatric population, such as a considerable impact on the state of anxiety. Moyer et al. [5] conducted a meta-analysis of randomized studies to test the effectiveness of massage therapy, and the results showed that massage therapy was superior in reducing anxiety and depression. Furthermore, several studies [14-16] have suggested that massage therapy is beneficial to children because it improves blood flow, normalizes function of the central nervous system, and reduces tissue stiffness.

For the treatment of acute diarrhoea in children, many clinical studies have reported beneficial effects of massage therapy. The theorized mechanism of massage therapy on acute diarrhoea is that it promotes gastrointestinal motility, regulates gastric acid secretion, and improves spontaneous bowel movements by stimulating acupuncture points [17-19], although the actual mechanism is still unclear. Considering no relevant systematic reviews have assessed the clinical effectiveness or the risk of paediatric massage therapy, in this study, a meta-analysis was conducted to assess the efficacy of paediatric massage for the treatment of acute diarrhoea in children.

\section{Methods}

The protocol of this study was registered in PROSPERO with a registration number CRD42017056523.

\section{Database and search strategies}

Relevant studies were searched in the following electronic databases: Cochrane Library, Web of Science, PubMed, Chinese Biomedical Literature Database, Chinese National Knowledge Infrastructure, Chinese Scientific Journal Database, and Wan-fang Database up to April 30, 2018. The following search terms were used: (massage OR tui na OR manipulation OR acupressure) AND (infant OR child OR baby OR paediatrics) AND (diarrhea OR diarrhoea) AND (randomized controlled trial). There were no language limitations.

\section{Inclusion criteria}

Included studies must all be randomized controlled trials (RCTs).

\section{Participants}

Paediatric patients who were diagnosed with acute diarrhoea. Acute diarrhoea is a disease defined as more stools than normal, and there are some changes in the stool traits, such as loose stool, or watery stool. Usually, course of the disease lasts less than 14 days.

\section{Interventions}

Interventions using massage therapy alone or combined with other non-pharmacological approaches were included. Interventions using massage therapy combined with pharmacological therapies, such as montmorillonite, were excluded.

\section{Comparators}

In the control groups, patients received pharmacotherapy.

\section{Outcomes}

Clinical effective rate was the primary outcome. Some other outcomes included clinical cure rate, and cure time.

\section{Exclusion criteria}

Patients with chronic diarrhoea were excluded. Studies lacking data for course of disease were excluded, because it is impossible to judge patients were in acute diarrhoea. Non-RCTs, unpublished or repeated literature, case studies, qualitative studies, and experience summaries were excluded.

\section{Data extraction and quality assessment}

Three reviewers (Gao, Jia, and Huang) independently extracted the data and conducted quality assessments. Statistical analysis was conducted using the RevMan 5.3 software, and risk of bias was assessed using the Cochrane tool. Any disagreement was resolved by discussions among the reviewers.

\section{Results \\ Description of included studies}

In this meta-analysis, 813 potentially eligible studies were identified, of which 787 were excluded, including 431 repeated publications, 145 irrelevant studies, 63 studies that combined pharmacological therapy in the intervention group, 110 studies that included patients with chronic diarrhoea, 17 studies that lacked data for the course of disease, 12 studies that did not meet inclusion criteria in the control group, and 9 studies that were not RCTs. Thus, a total of 26 studies [20-45] encompassing 2644 participants (1462 in the intervention group and 1182 in the control group) were included in the meta-analysis, and all were published in Chinese Journal Literature 
Databases. The screening process is summarized in a flow diagram (Fig. 1).

Details of the 26 studies are summarized in Table 1. All the children were under 5 years old, and disease course of the participants was less than 14 days. In the intervention group, massage therapy was used alone or combining with other non-pharmacological approaches to treat acute diarrhoea. There are many different treatment methods in the intervention group, and details of the interventions of the included studies are shown in additional file 1 . In general, these interventions can be classified into several categories. According to the book Massage [46] in China, there were some basic manipulations suggested for the treatment of acute diarrhoea, including pushing Pijing, Dachang upward, rubbing the abdomen, kneading navel, pushing Qijiegu upward, and Pinching spine. Almost all the studies used the basic massage treatment. Furthermore, in the aim of increasing efficacy, many physicians utilized acupressure (Pressing some acupuncture points), while some physicians performed an individual massage treatment, some other physicians used the acupuncture therapy. In the control group, all the studies used pharmacotherapy; fourteen studies [21, 23, 25, 26, 28-30, 34, 36-38, 42-44] used montmorillonite alone, while other studies used combined therapy.
Ten studies [21, 24, 26, 30, 34-36, 38, 40, 42] reported using standard of Diagnosis and treatment proposal for diarrhoea in China [47] for the definition of acute diarrhoea. This standard provides a diagnostic basis for acute diarrhoea, that is more stools than usual, some changes in the stool traits, and lasting less than 2 weeks. Seven studies [25, 28, $29,33,37,43,44]$ adopt a standard in traditional Chinese medicine [48], while other studies did not report any standards cited in the inclusion criteria. However, their definitions of acute diarrhoea in the inclusion criteria were similar to the standard of Diagnosis and treatment proposal for diarrhoea in China.

\section{Risk of bias}

The risk of bias was high in the included studies (Fig. 2). All the studies used randomization, but only seven [22, 30, 35, 38, 40,44,45] of these studies reported using an appropriate method of random sequence generation, while three $[25,31,36]$ of these studies reported using inappropriate methods. None of the studies described the method for allocation concealment and blinding of the outcome assessment. Most of the included studies had a high risk of performance bias,

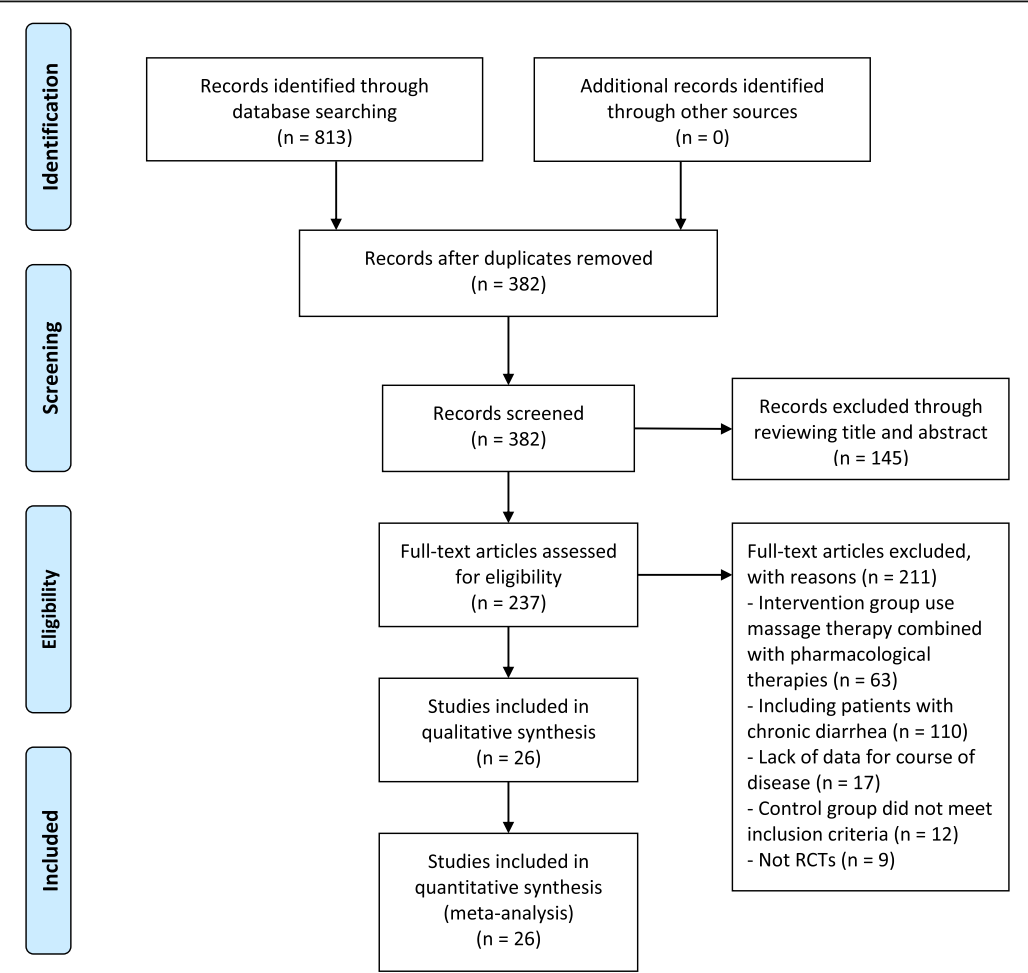

Fig. 1 PRISMA flow diagram 


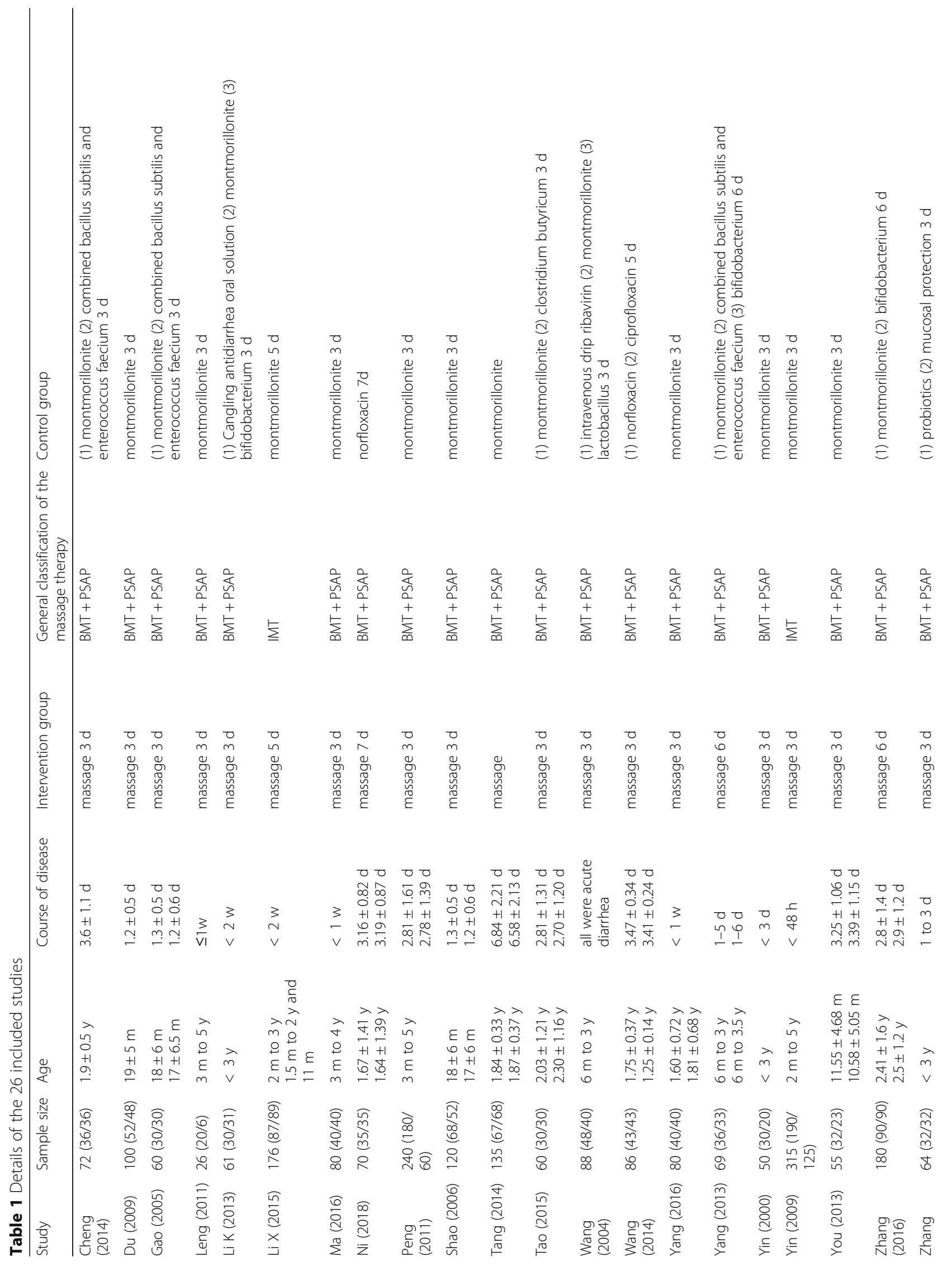




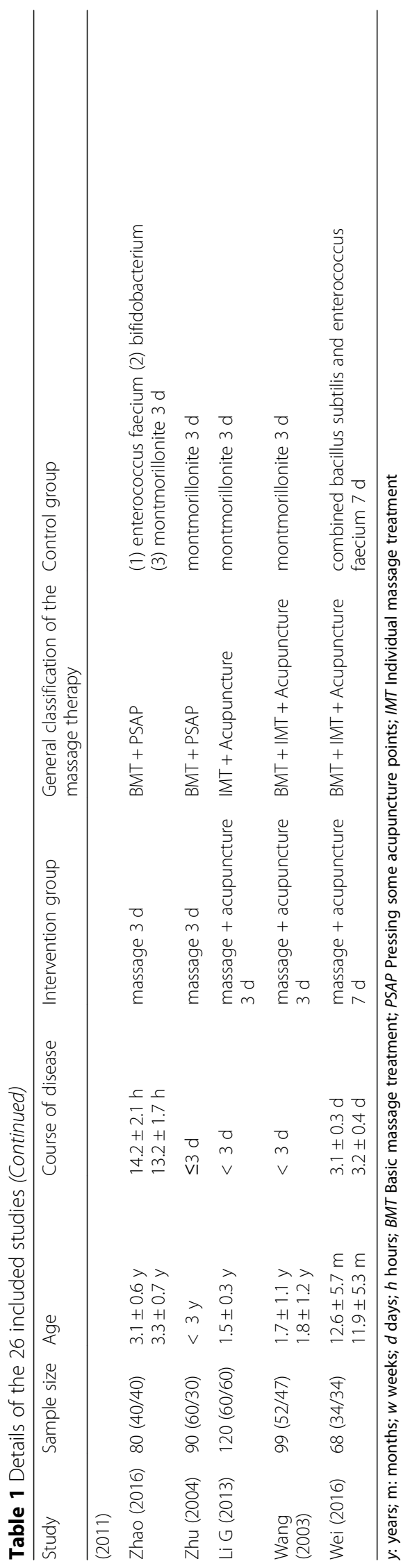


a

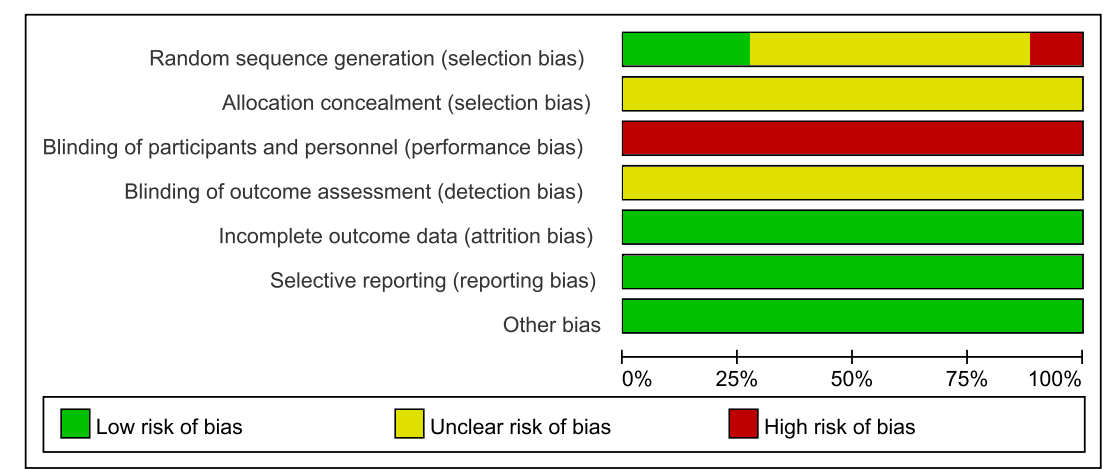

b

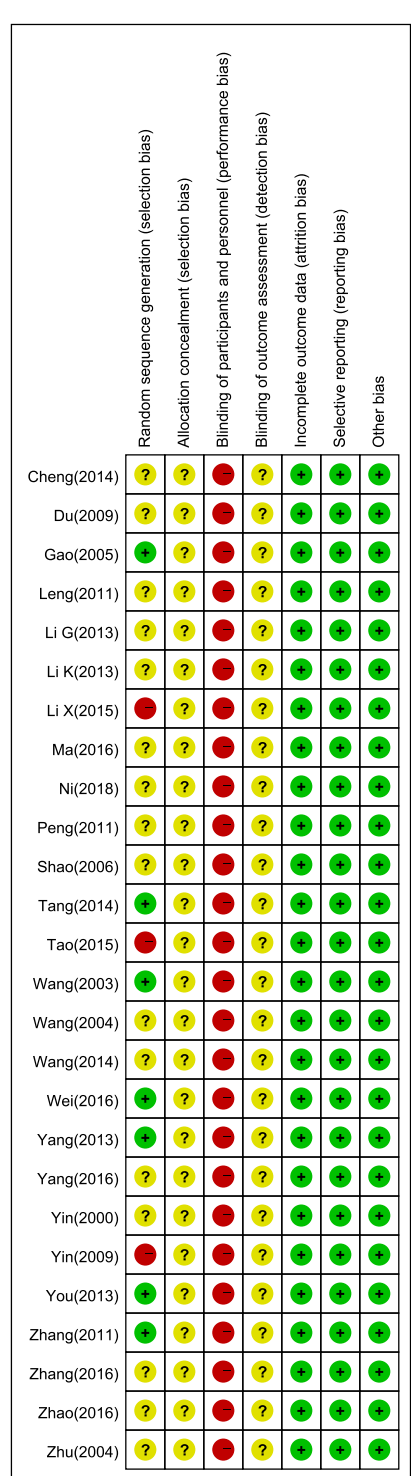

Fig. 2 Risk of bias graph: (a) risk of bias of all included studies; (b) Risk of bias summary

because both the physicians and the patients clearly knew which treatment was given.

\section{Outcome measurements Clinical effective rate}

According to the standard of Diagnosis and treatment proposal for diarrhoea in China [47], effective was defined as that there is significant improvement in stool traits after $72 \mathrm{~h}$ of treatment, and the frequency of stools reduced by $50 \%$. Only four of all studies did not use this standard to define effective. They adopt a similar method, the difference is that two studies [35, 39] did the evaluation after 6 days of treatment, while two studies [27, 45] did the evaluation after 7 days of treatment.

Twenty-two studies showed that massage therapy had a higher clinical effective rate compared with pharmacotherapy. Since high heterogeneity was observed in the meta-analysis $\left(\mathrm{I}^{2}=64 \%\right.$, which is higher than $\left.50 \%\right)$, a random-effects model was used to calculate the pooled estimation with analysis of dichotomous data using relative risk (RR), including 95\% confidence intervals (CIs). The meta-analysis showed favourable effects of massage therapy in clinical effective rate $(n=2213, \mathrm{RR}=1.20$, 95\% CI: 1.14 to $1.27, P<0.01)$ compared with the control group (Fig. 3). A subgroup analysis was conducted for massage therapy alone and massage combined with 


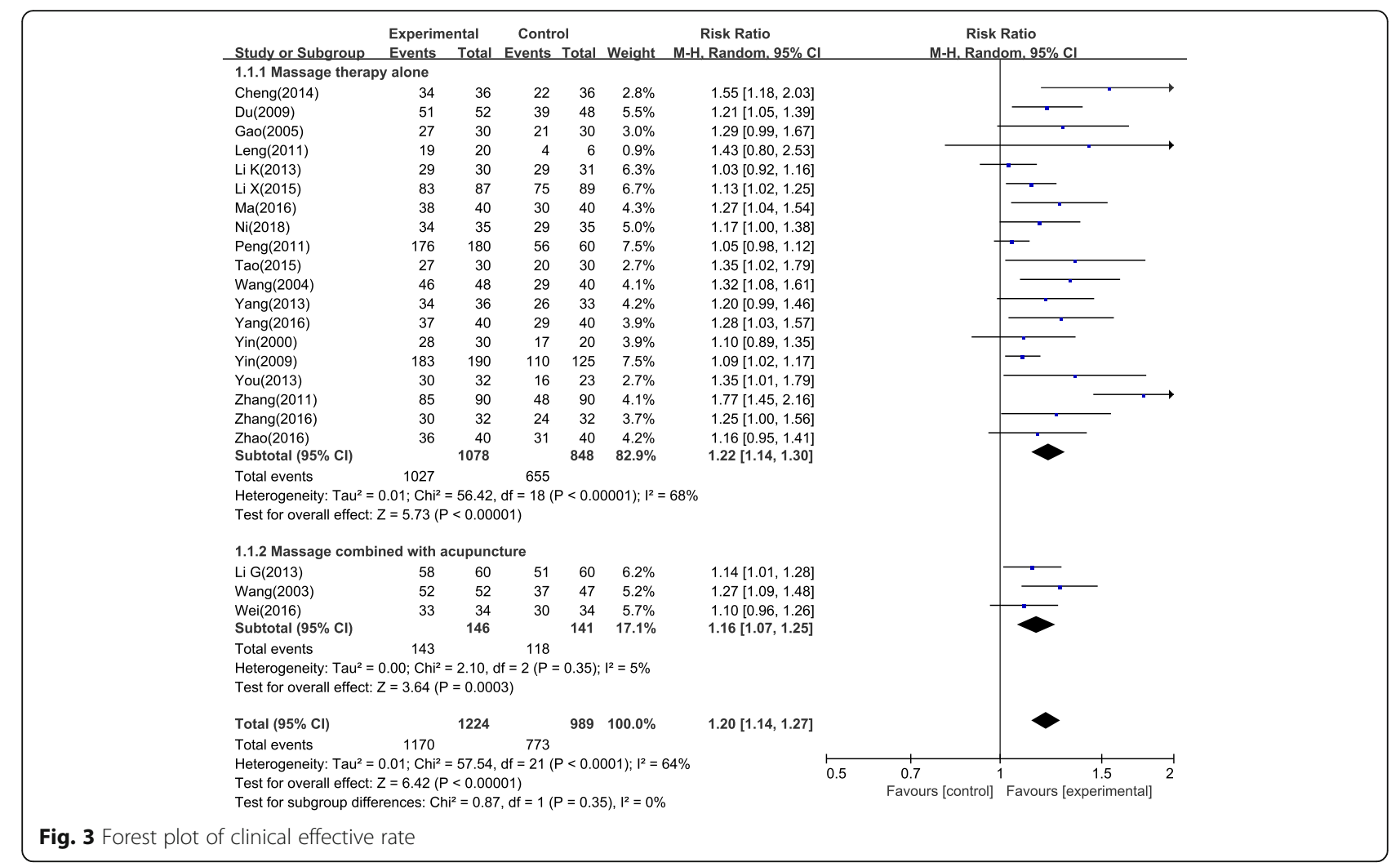

acupuncture. The results showed high heterogeneity in the subgroup massage therapy alone, with $\mathrm{I}^{2}=68 \%$. While there was low heterogeneity for subgroup differences, with $\mathrm{I}^{2}=0 \%$.

The reason for the high heterogeneity in the subgroup massage therapy alone may be due to different pharmacotherapies in the control group. Since several studies used montmorillonite alone in the control group, a subgroup analysis was also performed for montmorillonite alone and combined therapy, as shown in Fig. 4. The subgroup of montmorillonite had 1122 patients, with $\mathrm{RR}=1.13,95 \%$ CI: 1.07 to $1.20, P<0.01$. The subgroup with combined therapy had 804 patients, with $\mathrm{RR}=1.28,95 \%$ CI: 1.13 to $1.45, \mathrm{P}<0.01$. Heterogeneity in the subgroup montmorillonite was low $\left(\mathrm{I}^{2}=32 \%\right)$, while heterogeneity in the subgroup of combined therapy was high $\left(\mathrm{I}^{2}=74 \%\right)$, which resulted in a significant difference between these two subgroups, with $\mathrm{I}^{2}=68.5 \%$. Thus, it can be concluded that the differences in the pharmacotherapies in the control group was a main reason for the high heterogeneity.

\section{Clinical cure rate}

Cure was defined as that the stool traits and frequency returned to normal within $72 \mathrm{~h}$ of treatment. Three studies [29, 30, 42] showed that massage therapy had a higher clinical cure rate compared with pharmacotherapy $\left(n=345, \mathrm{RR}=1.37,95 \% \mathrm{CI}: 1.19\right.$ to $\left.1.57, \mathrm{I}^{2}=0 \%\right)$, as shown in Fig. 5.

\section{Cure time}

Cure time was defined as the length of treatment time spent before the patient is completely cured. Six studies [21, 30, 33, 35, 38, 45] reported data on cure time. The meta-analysis of cure time shows a mean difference (MD) of -0.77 (95\% CI: -0.89 to 0.64 ) with a low heterogeneity $\left(\mathrm{I}^{2}=37 \%\right)$, as shown in Fig. 6, which means that the participants receiving massage therapy had shorter cure time than those with pharmacotherapy.

\section{Discussion}

In TCM, paediatric massage therapy takes into consideration each child's individual physical development and has been found to have many benefits through manipulation on acupoints, such as improving digestive system [49], promoting mental and physical health [11], and increasing body weight in premature infants [50]. However, massage therapy should be carried out by medical staff with enough training, for inaccurate 


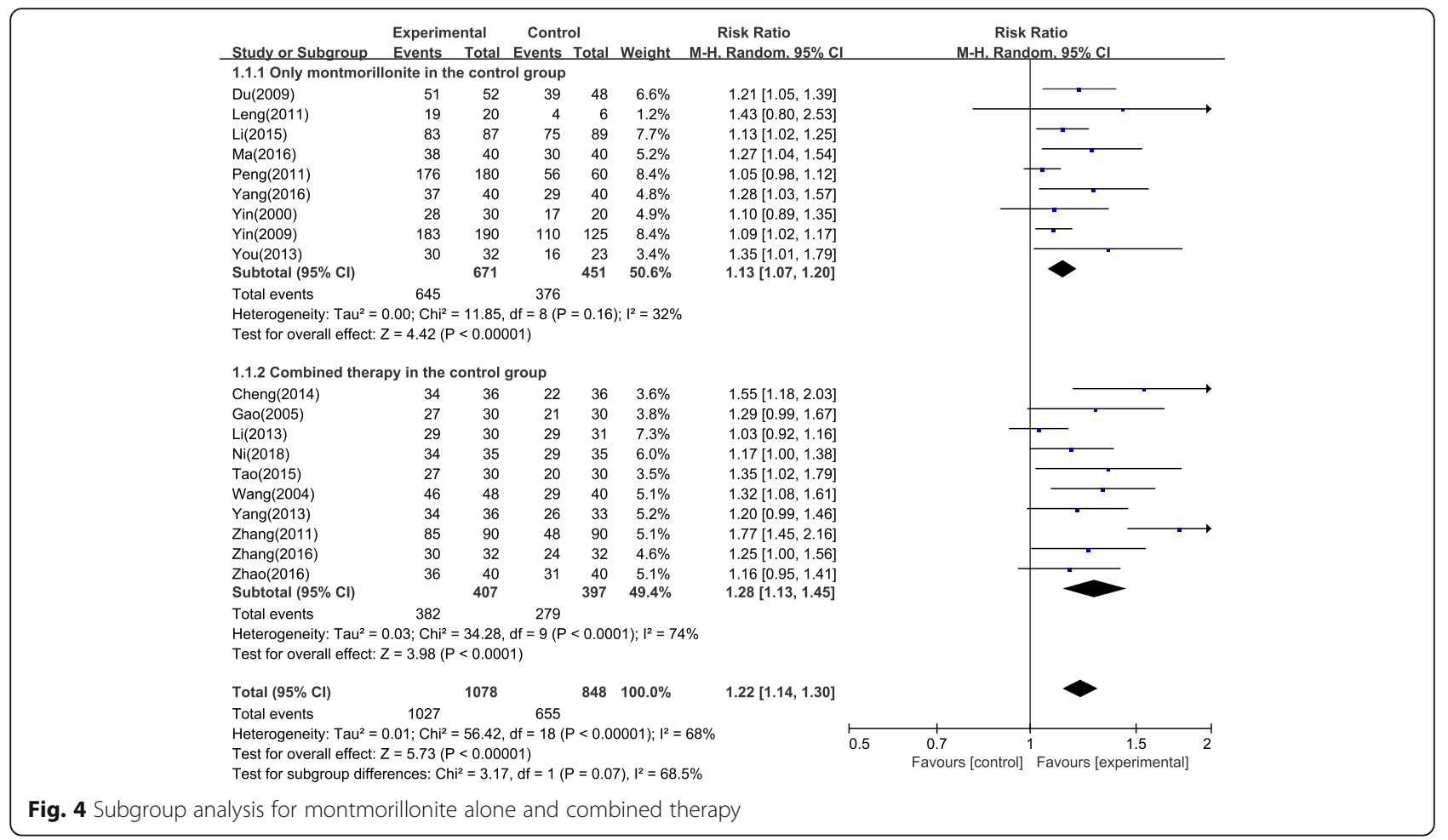

treatment may reduce the clinical effects. As a complementary and alternative medicine, massage therapy benefits the body in a way that is quick, easy, and inexpensive. It was reported that paediatric massage therapy has been used for treating diseases in children for thousands of years [51]. In recent years, many physicians have reported that paediatric massage therapy can effectively improve the function of the spleen and stomach by stimulating some specific acupoints [19, 49]. However, clinical effectiveness and risk have not been systematically assessed. This study reported a meta-analysis of massage therapy for the treatment of acute diarrhoea in children.

In the treatment of acute diarrhoea in children, massage therapy acts on the on meridians and acupuncture points, activating the flow of Qi and nourishing spleen and stomach. As a result, the function of the digestive system is improved. In addition, several studies have reported that massage therapy has a positive effect on emotional state of children $[52,53]$, and as we know, a good emotional state has benefits for improving immune function. It is reasonable to think that part of the rationale for massage therapy to address acute diarrhoea is improving emotional state in paediatric patients.

High heterogeneity was found in clinical effective rate, with $\mathrm{I}^{2}=64 \%$. Reasons may include different pharmacotherapies used in different control groups. For example, $\mathrm{Du}$ [21] used montmorillonite in the control group for the treatment of diarrhoea, while Zhang [40] used probiotics and mucosal protection. These different therapies make the efficacy of massage therapy hard to assess. We conducted a subgroup analysis for montmorillonite alone and combined therapy. It was shown that heterogeneity in the subgroup montmorillonite was low $\left(\mathrm{I}^{2}=32 \%\right)$,

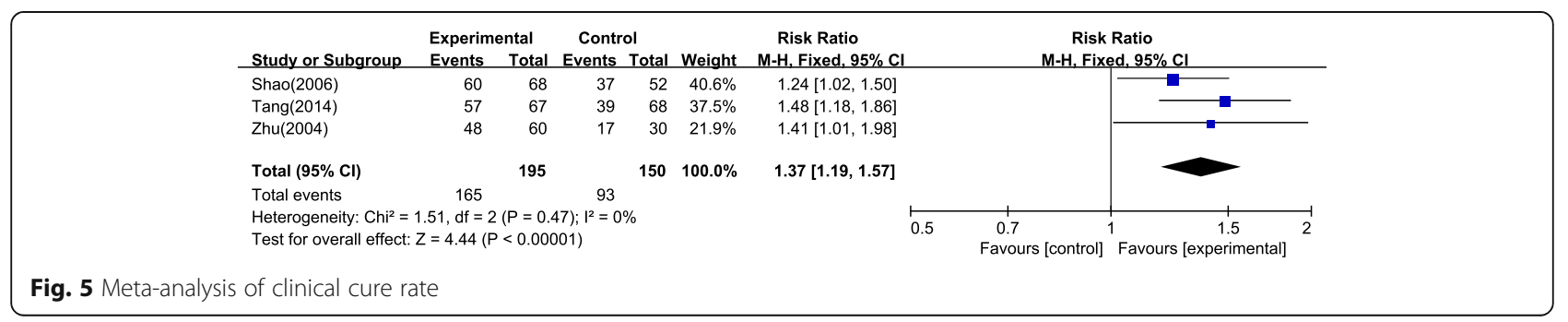




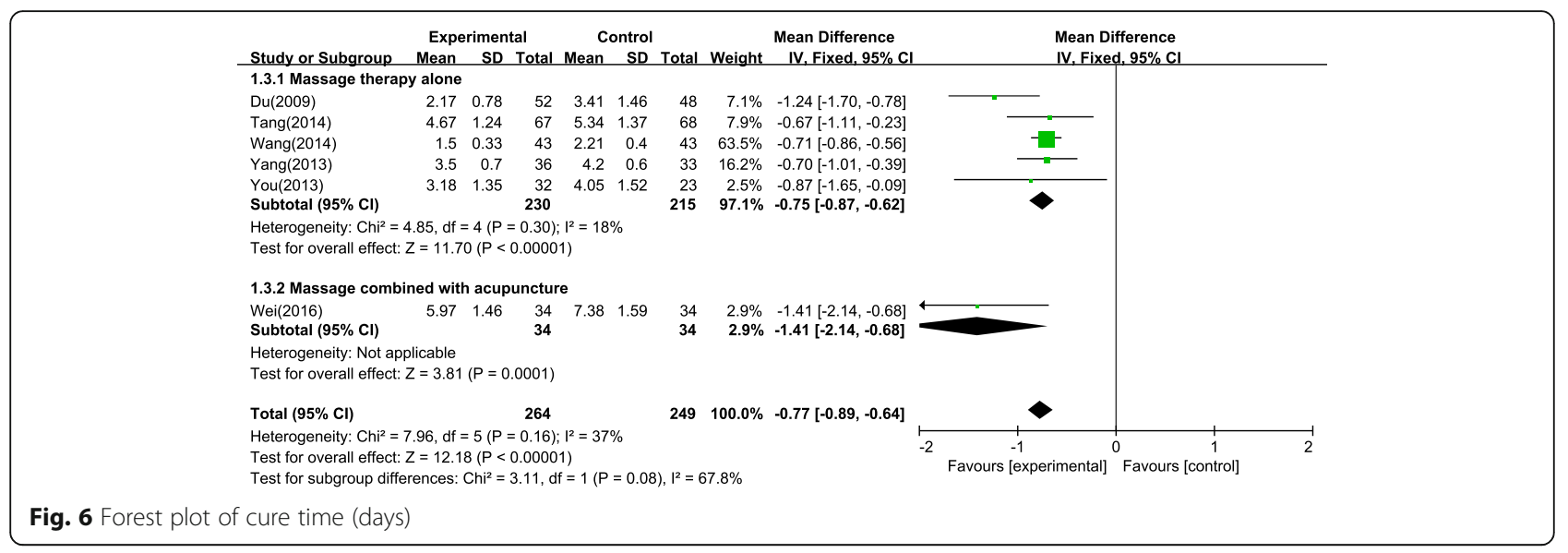

while heterogeneity in the subgroup combined therapy was high $\left(\mathrm{I}^{2}=74 \%\right)$, resulting in a significant difference between these two subgroups. It can be concluded that the differences in pharmacotherapies in the control group was a main reason for the high heterogeneity. Second,

In addition, another reason for high heterogeneity was that different massage techniques were used by different TCM physicians (as shown in additional file 1). In these studies, some basic manipulations were utilized by all the physicians. However, more manipulations were conducted in different ways, massaged parts of body, manipulation order, and manipulation frequency are usually different. Furthermore, diarrhoea is classified into different types by different TCM physicians, such as cold damp type, spleen deficiency type, and damp hot type; thus, different massage therapy techniques were applied to different types of diarrhoea.

The methodological quality for this finding was low because of high risk of bias. There are several limitations in this systematic review. First, for most of the included studies, the methods for randomization, allocation concealment, and blinding were not reported clearly. Due to the characteristics of TCM, both the physicians and the patients clearly knew which treatment was been given, making blinding methods difficult. Second, in the 26 included studies, only 8 studies had sample sizes greater than 100 trials; small sample sizes in most studies made it hard to draw a meaningful conclusion. Third, clinical effective rate was the main outcome measurement for most studies, and thus, bias from the physicians might decrease reliability and validity of the studies. Fourth, limited information about adverse effects was reported by the included studies; therefore, conclusions on the safety of massage therapy on treatment of acute diarrhoea should be seriously considered. Fifth, all the studies were conducted in China, which may limit generalization of the findings. Considering the limitations in this meta-analysis, it is strongly recommended that more rigorous RCTs with large sample sizes should be used to further evaluate the clinical efficacy and adverse effects of paediatric massage in treating acute diarrhoea in children.

\section{Conclusions}

A total of 26 studies encompassing 2644 patients were included in this meta-analysis that compared paediatric massage and pharmacotherapy for treating acute diarrhoea in children. The results of the meta-analysis suggest that massage therapy was superior to pharmacotherapy. However, the studies analysed to date are of relatively low quality. More rigorous RCTs with large sample sizes are recommended to further evaluate the clinical efficacy and adverse effects of paediatric massage in treating acute diarrhoea in children.

\section{Additional file}

Additional file 1: Details of the massage therapy. Details of the interventions of the included studies. (DOCX $19 \mathrm{~kb}$ )

\section{Abbreviations}

RCTs: Randomized controlled trials; TCM: Traditional Chinese medicine

Funding

This study was funded by the National Natural Science Foundation of China (Grant No. 81373770).

Availability of data and materials

All data generated or analysed during this study are included in this published article and its supplementary information files.

Authors' contributions

LG contributed to conception, acquisition, analysis, and interpretation; CJ contributed to conception, design, and analysis; $\mathrm{HH}$ contributed to conception, and analysis. All authors drafted manuscript, revised manuscript, gave final approval, agreed to be accountable for all aspects of work ensuring integrity and accuracy. 
Ethics approval and consent to participate

Not applicable.

\section{Consent for publication}

Not applicable.

\section{Competing interests}

The authors declare that they have no competing interests.

\section{Publisher's Note}

Springer Nature remains neutral with regard to jurisdictional claims in published maps and institutional affiliations.

Received: 5 June 2018 Accepted: 12 September 2018 Published online: 18 September 2018

\section{References}

1. Vos T, Barber RM, Bell B, Bertozzi-Villa A, Biryukov S, et al. Global, regional, and national incidence, prevalence, and years lived with disability for 301 acute and chronic diseases and injuries in 188 countries, 1990-2013: a systematic analysis for the global burden of disease study 2013. Lancet. 2015:386(9995):743-800

2. Caramia G, Silvi S, Verdenelli MC, Coman MM. Treatment of acute diarrhoea: past and now. Int J Enteric Pathog. 2015;3(4):e28612.

3. Lamberti LM, Walker CLF, Chan KY, Jian WY, Black RE. Oral zinc supplementation for the treatment of acute diarrhea in children: a systematic review and meta-analysis. Nutrients. 2013;5(11):4715-40.

4. Li ST, Grossman DC, Cummings P. Loperamide therapy for acute diarrhea in children: systematic review and meta-analysis. PLoS Med. 2007;4(3):e98.

5. Moyer CA, Rounds J, Hannum JW. A meta-analysis of massage therapy research. Psychol Bull. 2004;130(1):3-18.

6. Furlan AD, Giraldo M, Baskwill A, Irvin E, Imamura M. Massage for low-back pain. Cochrane Database Syst Rev. 2015;9:CD001929.

7. Lee MS, Kim JI, Ernst E. Massage therapy for children with autism spectrum disorders: a systematic review. J Clin Psychiat. 2011;72(3):406-11.

8. Beider S, Mahrer NE, Gold Jl. Pediatric massage therapy: an overview for clinicians. Pediatr Clin N Am. 2007;54(6):1025-41.

9. Lämås K, Häger C, Lindgren L, Wester P, Brulin C. Does touch massage facilitate recovery after stroke? A study protocol of a randomized controlled trial. BMC Complem Altern M. 2015;16:50.

10. Kania-Richmond A, Reece BF, Suter E, Verhoef MJ. The professional role of massage therapists in patient care in Canadian urban hospitals - a mixed methods study. BMC Complem Altern M. 2015;15:20.

11. Bennett C, Underdown A, Barlow J. Massage for promoting mental and physical health in typically developing infants under the age of six months. Cochrane Database Syst Rev. 2013;4:CD005038

12. Vickers A, Ohlsson A, Lacy JB, Horsley A. Massage for promoting growth and development of preterm and/or low birth-weight infants. Cochrane Database Syst Rev. 2000;41(2):CD000390.

13. Beider S, Moyer CA. Randomized controlled trials of pediatric massage: a review. Evid Based Complement Alternat Med. 2007;4(1):23-34.

14. Diego MA, Field T, Hernandezreif M. Vagal activity, gastric motility, and weight gain in massaged preterm neonates. J Pediatr. 2005;147(1):50-5.

15. Mcgrath JM. Touch and massage in the newborn period: effects on biomarkers and brain development. J Perinat Neonat Nur. 2009;23(4):304-6.

16. Procianoy RS, Mendes EW, Silveira RC. Massage therapy improves neurodevelopment outcome at two years corrected age for very low birth weight infants. Early Hum Dev. 2010;86(1):7-11.

17. Hui Z, Ying L, Wei Z, Fang Z, Zhou SY, et al. Electroacupuncture for patients with diarrhea-predominant irritable bowel syndrome or functional diarrhea: a randomized controlled trial. Medicine. 2016;95(24):e3884.

18. Qin Z, Li B, Wu J, Tian J, Xie S, et al. Acupuncture for chronic diarrhea in adults:protocol for a systematic review. Medicine. 2017;96(4):e5952.

19. Wei R, Chen Z, Yan J. "Four-step massage" in treating childhood spleen-deficiency diarrhea. Journal of Acupuncture and Tuina Science. 2006:4(5):271-3.

20. Cheng G. Clinical study of massage in the treatment of 36 cases of pediatric noninfectious diarrhea. J North Phar. 2014;11(7):83.

21. Du L. Traditional massage therapy for treating 52 cases of infantile autumn diarrhea. Chinese Journal of Ethnomedicine and Ethnopharmacy. 2009;24:111-3.
22. Gao G. Clinical observation of massage therapy for treating children rotaviral enteritis. Clinical Journal of Traditional Chinese Medicine. 2005;17(3):267-8

23. Leng L, Zhang J, Wang D, Chen Z, Xing F, et al. Clinical effect observation of massage therapy for treating 20 cases of children cold diarrhea. Nei Mongol Journal of Traditional Chinese Medicine. 2011:8:13.

24. Li K, Xie Y, Ying X. Tuina therapy for treating infant damp-heat diarrhea. Journal of Beijing University of Traditional Chinese Medicine (Clinical Medicine). 2013;20(5):26-30.

25. Li X, Yan Y, Dian Y. Clinical effective observation on treating diarrhea in the acute phase in infants by massage. Clinical Journal of Chinese Medicine. 2015;7(35):22-3.

26. Ma B. Clinical analysis of anterograde and retrograde massage therapy for treatment of children rotaviral enteritis. Modern Health. 2016;18:86.

27. Ni Y. Clinical efficacy observation of massage therapy on acute diarrhea in children. Diet Health. 2018;5(6):84-5.

28. Peng $Y$, Leng $L$, Chen $Z$, Zhang J, Wang $D$, et al. Acute infantile diarrhea treated with infantile Tuina: a multicentre randomized controlled trial. Chinese Acupuncture \& Moxibustion. 2011;31(12):1116-20.

29. Shao X, Wei G, Liu Y. Clinical observation of Liu massage therapy for treatment of 68 cases of children rotaviral enteritis. New Journal of Traditional Chinese Medicine. 2006;38(3):67-8.

30. Tang $Y$, Shang $Q$, Yan X. Clinical studies in children with diarrhea by tonifying spleen qi manipulation. China Journal of Chinese Medicine. 2014;29(3):444-5.

31. Tao H, Li Z, Ling X. Clinical efficacy of massage in treatment of infantile indigestion diarrhea caused by improper diet: a report of 30 cases. Journal of Anhui University of Chinese Medicine. 2015;34(6):33-5.

32. Wang $T$, Jiang W. Massage therapy for treatment of 48 cases of children rotaviral enteritis. Shaanxi Journal of Traditional Chinese Medicine. 2004;5:445.

33. Wang Y. Clinical observation of massage therapy for treating 43 cases of children diarrhea. Chinese Journal of Ethnomedicine and Ethnopharmacy. 2014;21:33.

34. Yang J, Yang T. Clinical study of anterograde and retrograde massage therapy for treatment of children rotaviral enteritis. Practical Clinical Journal of Integrated Traditional Chinese and Western Medicine. 2016;16(2):36-7.

35. Yang $Y$, Zhang $Y$. Clinical study of massage in the treatment of pediatric noninfectious diarrhea. China Journal of Chinese Medicine. 2013;28(11):1763-5.

36. Yin C, Xiao Z. Observation and care of massage therapy for treatment of children diarrhea. Today Nurse. 2009;9:61-3.

37. Yin $\mathrm{M}$, Chen $\mathrm{X}$, Han $\mathrm{X}$, Wu Y. Massage therapy for treating 30 cases of children rotaviral enteritis. Journal of Nanjing University of Traditional Chinese Medicine (Natural Science). 2000;16(1):40-1.

38. You X. Massage therapy for treating 32 cases of infantile diarrhea. Chinese Pediatrics of Integrated Traditional and Western Medicine. 2013;5(5):423-5.

39. Zhang J. Clinical effect study of massage therapy in combination with diet instruction for treating children diarrhea. Guangming Journal of Chinese Medicine. 2016;31(14):2078-80.

40. Zhang Z, Shen $H$, Wang $X$. Clinical efficacy of transporting massage and stopping diarrhea in the treatment of children rotavirus enteritis. Liaoning Journal of Traditional Chinese Medicine. 2011;38(5):967-8.

41. Zhao W, Song J, Junfeng S. Syndrome differentiation of traditional Chinese medicine massage therapy in clinical application in the treatment of infantile diarrhea. Contemporary Medical Symposium. 2016;14(8):24-5.

42. Zhu Q, Hou D, Zheng Y. Clinical observation of massage therapy in combination with finger pressing for treating children diarrhea. Hubei Journal of Traditional Chinese Medicine. 2004;26(5):49-50.

43. Li G. Treatment of 60 cases of autumn diarrhea in children with acupuncture and massage. Henan Traditional Chinese Medicine. 2013; 33(10):1690-1.

44. Wang X, Bian D, Ma Q. Clinical observation on treating autumn diarrhea in children with acupuncture and massage. Liaoning Journal of Traditional Chinese Medicine. 2003;30(6):492-3.

45. Wei H. Observation on the therapeutic effect of treating children with diarrhea with acupuncture and massage. Journal of Clinical Acupuncture and Moxibustion. 2016;32(7):33-4.

46. Yu D. Massage Science. Shanghai: Shanghai Science and Technology Press; 1995.

47. Fang $H$, Wei $C$, Duan S, Dong $X$, Dong $Y$, et al. Diagnosis and treatment proposal for diarrhea in China. Chinese Journal of Practical Pediatrics. 1998; 13(6):381-4 
48. State Administration of Traditional Chinese Medicine: Criteria of diagnosis and therapeutic effect of paediatric diseases and syndromes in traditional Chinese medicine. 1994.

49. Xia $Q$, Feng $Z$, Ping $C$. Evaluating the efficacy of Tui $\mathrm{Na}$ in treatment of childhood anorexia: a meta-analysis. Altern Ther Health Med. 2014;20(5):45-52.

50. Chen L, Su Y, Su C, Lin H, Kuo H. Acupressure and meridian massage: combined effects on increasing body weight in premature infants. J Clin Nurs. 2008;17(9):1174-81.

51. Badr LK, Abdallah B, Kahale L. A meta-analysis of preterm infant massage: an ancient practice with contemporary applications. Mcn the American Journal of Maternal Child Nursing. 2015;40(6):341-7.

52. Suresh S, Wang S, Porfyris S, Kamasinski-SOL R, Steinhorn D. Massage therapy in outpatient pediatric chronic pain patients: do they facilitate significant reductions in levels of distress, pain, tension, discomfort, and mood alterations? Pediatr Anesth. 2008;18(9):884-7.

53. Kemper K, Shannon S. Complementary and alternative medicine therapies to promote healthy moods. Pediatr Clin N Am. 2007;54(6):901-26.

Ready to submit your research? Choose BMC and benefit from:

- fast, convenient online submission

- thorough peer review by experienced researchers in your field

- rapid publication on acceptance

- support for research data, including large and complex data types

- gold Open Access which fosters wider collaboration and increased citations

- maximum visibility for your research: over $100 \mathrm{M}$ website views per year

At $\mathrm{BMC}$, research is always in progress.

Learn more biomedcentral.com/submissions 